SHORT REPORT

\title{
Pulmonary tumour microembolism clinically mimicking alveolitis
}

\author{
A W I Lo, G M K Tse, W C W Chu, A B W Chan
}

A 56 year old man with previously unsuspected recurrence of squamous cell carcinoma of the oesophagus presented with dyspnoea. Bronchoscopy and computed tomography suggested bronchopneumonic changes with an infectious cause. He suffered a rapidly deteriorating course and died despite active treatment, including antibiotics and mechanical ventilation. Necropsy revealed a florid pulmonary tumour microembolism mimicking alveolitis. No bronchopneumonia was seen. The emboli arose from loosely attached tumour vegetations in the tricuspid valve. In a patient with known malignancy, tumour microembolism should be considered as an uncommon cause of rapid respiratory failure, refractory to antibiotic treatment.

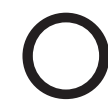
esophageal carcinoma is relatively common in Japan and Southern China. ${ }^{1}$ Because of the anatomical position of the oesophagus, complete resection of the tumour is difficult. Prognosis is generally poor and the five year recurrence rate is $10 \%$. We describe a case of unsuspected recurrence of oesophageal carcinoma, resulting in florid pulmonary tumour microembolism and rapidly progressive fatal respiratory failure.

\section{CASE REPORT}

A 56 year old man with squamous cell carcinoma of the oesophagus was treated with chemotherapy and radiotherapy three years previously. The tumour initially resolved but recurred one year later. He underwent a three staged oesophagectomy and was disease free for two years. He presented to our hospital because of worsening shortness of breath for one week. He was afebrile and initial microbiological investigations were all negative. High resolution computed tomography (CT) of the lung revealed patchy areas of mainly peripheral alveolar opacification in the mid and lower zones bilaterally. The CT changes were suggestive of an infective or idiopathic alveolitis (fig lA). The impression from bronchoscopy was that of pneumonia. Echocardiography showed mild tricuspid regurgitation, with right ventricular dilatation. Despite aggressive antibiotics and mechanical respiratory support, his condition worsened and he developed respiratory failure. The patient succumbed rapidly the next day. Necropsy revealed "consolidations" of both lungs, especially the lower lobes. Friable vegetations were also seen loosely attached to the tricuspid valves (fig 1B). Large emboli were not detected in the major pulmonary vessels. Distorted anatomy with fibrosis around the duodenum, in keeping with previous surgery, was also noted.

Microscopy revealed that the tricuspid vegetation consisted of fibrin and squamous carcinoma cells (fig 1C), with myocardial sparing. The lung showed tumour plugs in nearly all the medium to small sized pulmonary arteries, in addition to the capillaries, without spreading into the adjacent alveolar spaces. The arterial wall appeared to be normal. There was no intimal thickening. Pneumonic changes were not apparent (fig 1D).

In the fibrotic adhesion around the duodenum, nests of squamous carcinoma cells were seen infiltrating the submucosa without disrupting the duodenal mucosa. The tumour infiltrated through the muscularis propria, involving the pancreas and surrounding tissues, including nerves and ganglia. Lymphovascular infiltration was noted.

\section{DISCUSSION}

Pulmonary tumour embolism is not uncommon at necropsy, ranging from $2.4 \%$ to $26 \%$, ${ }^{2}$ but is rarely recognised clinically. As many as seven of 16 patients with oesophageal carcinoma ${ }^{3}$ had pulmonary tumour embolism, although only half of these patients had a history of respiratory distress. Large saddle-type tumour emboli result in a rapidly fatal course, ${ }^{4}$ whereas microscopic tumour emboli may be more subtle. $^{2}$

In our present case, there was extensive pulmonary tumour microemboli, resulting in subacute cor pulmonale and respiratory failure. These tumour microemboli probably arose from the detachment of the loosely attached tumour vegetation on the tricuspid valve cusps, and the lodgement of these tumour emboli in the pulmonary vasculature haematogenously. The reason for tumour vegetation formation on the tricuspid valve was not apparent. There were no valvular structural defects, and no history of rheumatic diseases was noted. Because no tumour was seen in the inferior vena cava, a haematogenous origin of the vegetation was more likely than contiguous spread. Minimal reaction of the vessel wall to the tumour emboli suggested a relatively short and recent history of the embolisation event.

Radiological diagnosis of pulmonary tumour microemboli is difficult. CT is helpful when there is a high index of suspicion. Spiral CT can detect emboli within proximal arteries of less than fifth or sixth generation as intraluminal filling defects. It is worth noting that metastasis of squamous cell carcinoma typically results in variably sized round nodules and cavitations. Cytology of an aspirated specimen from the pulmonary artery might be useful to establish the diagnosis. ${ }^{5}$

\section{"Radiological diagnosis of pulmonary tumour microem- boli is difficult"}

In our case, the trapping of tumour cells appears to be solely a mechanical sieve effect. Tissue reactions and changes in the arterial walls were lacking. This is in contrast to a condition known as "carcinomatous arteriopathy", in which the presence of tumour cells in the pulmonary vasculature caused myxoid fibrocellular proliferation with small lumens after revascularisation. ${ }^{6}$ 


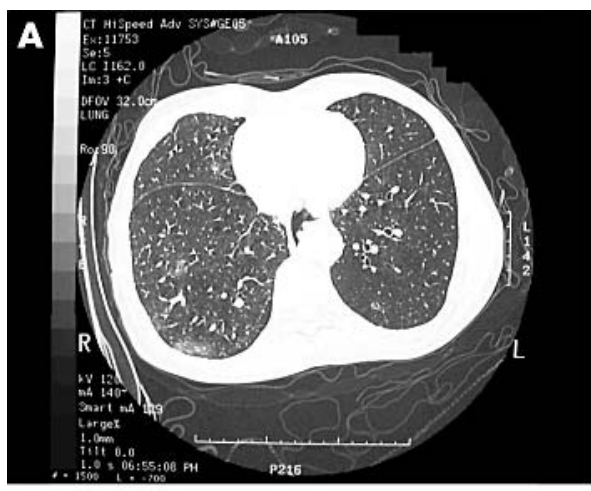

B
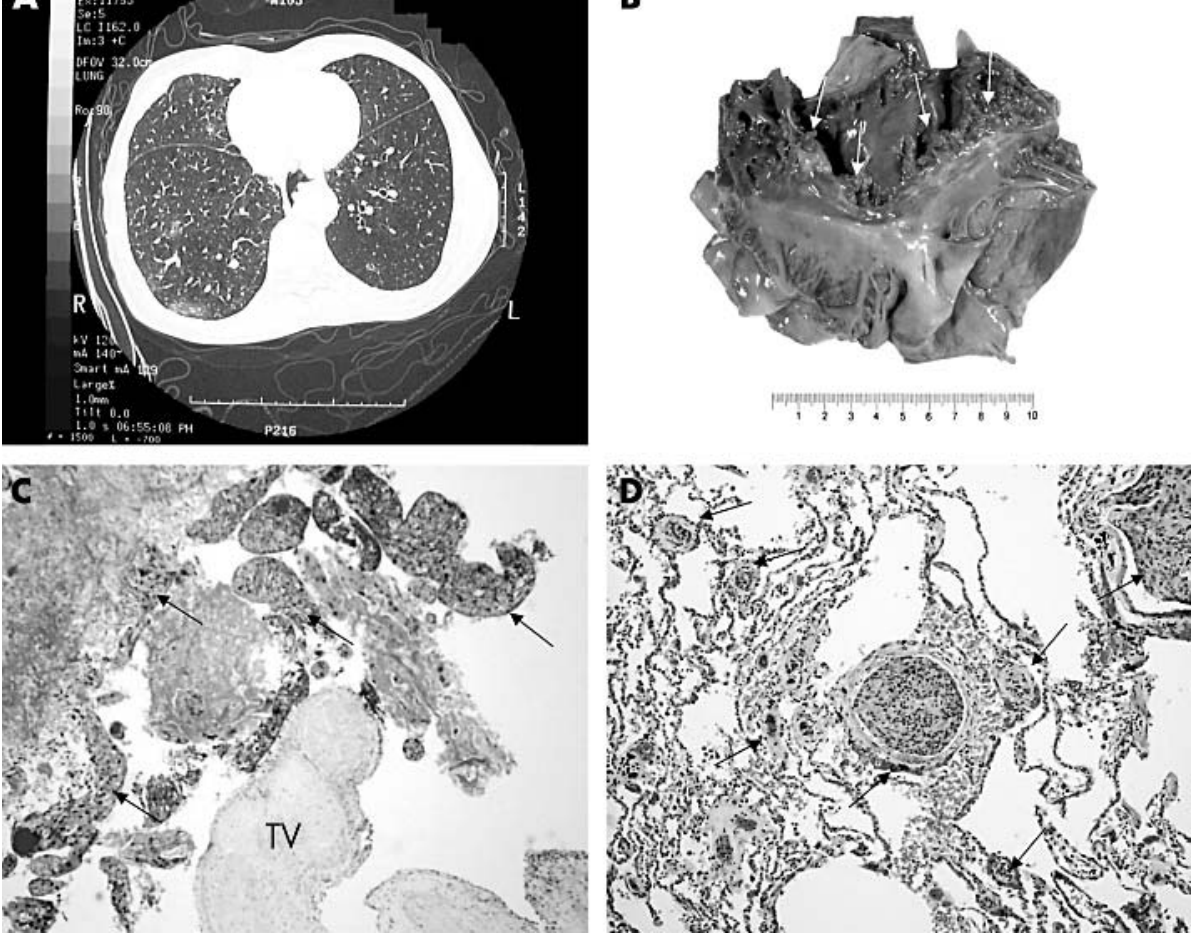

Figure 1 (A) High resolution computed tomography of the thorax showing a characteristic diffuse alveolar pattern suggestive of alveolitis. (B) Gross appearance of the right chambers of the heart showing the vegetations (arrows) on the tricuspid valve. (C) Haematoxylin and eosin (HE) stained section of the tricuspid valve (TV) with the loosely attached vegetation. The vegetation was composed of fibrin and tumour cells of squamous cell carcinoma (arrows) (original magnification, $\times 100)(D) \mathrm{HE}$ stained section of the lung showing tumour cells plugging the small to medium sized arteries and capillaries (arrows) without eliciting an intimal proliferative response. Tumour invasion into the lung parenchyma was not seen (original magnification, $\times 100$ ).
We report a case of extensive pulmonary tumour microemboli resulting in the rapid onset of respiratory failure in a patient with unsuspected recurrence of oesophageal squamous cell carcinoma. The imaging of the lungs was indistinguishable from infective alveolitis, rendering a clinical differential diagnosis difficult. The source of the tumour emboli was a tricuspid valve tumour vegetation, which is also an uncommon presentation of a recurrent oesophageal squamous cell carcinoma, particularly because there was no clinical evidence of recurrence at other sites. Tumour microemboli in the pulmonary vasculature has to be considered as a possible cause of respiratory failure in

\section{Take home messages}

- We report a case of extensive pulmonary tumour microemboli, which resulted in the rapid onset of respiratory failure, in a patient with unsuspected recurrence of oesophageal squamous cell carcinoma

- Imaging suggested bronchopneumonic changes with an infectious cause and a clinical differential diagnosis was difficult

- Tumour microemboli in the pulmonary vasculature should be considered as a possible cause of respiratory failure in patients with a history of squamous cell carcinoma, particularly of the upper aerodigestive tract patients with a history of squamous cell carcinoma, particularly of the upper aerodigestive tract.

\section{Authors' affiliations}

A W I Lo, G M K Tse, A B W Chan, Department of Anatomical and Cellular Pathology, The Chinese University of Hong Kong, Prince of Wales Hospital, Shatin, NT, Hong Kong SAR, China

W C W Chu, Department of Diagnostic Radiology and Organ Imaging, The Chinese University of Hong Kong

Correspondence to: Dr A B W Chan, Department of Anatomical and Cellular Pathology, Chinese University of Hong Kong, Prince of Wales Hospital, Ngan Shing Street, Shatin, NT, Hong Kong SAR, China; amychan@cuhk.edu.hk

Accepted for publication 15 May 2003

\section{REFERENCES}

1 Gabbert HE, Shimoda T, Hainaut $P$, et al. Squamous cell carcinoma of the oesophagus. In: Hamilton SR, Aaltonen LA, eds. World Health Organisation classification of tumours: pathology and genetics of tumours of the digestive system. Lyon: IARC Press, 2000:11-19.

2 Hibbert M, Braude S. Tumour microembolism presenting as "primary pulmonary hypertension". Thorax 1997:52:1016-17.

3 Soares FA, Landell GA, de Oliveira JA. Pulmonary tumour embolism from squamous cell carcinoma of the oesophagus. Eur J Cancer 1991;27:495-8.

4 Ha JW, Kim SK, Chang BC. Pulmonary tumour embolism. Lancet 2002;359:2158.

5 Seo JB, Im JG, Goo JM, et al. Atypical pulmonary metastases: spectrum of radiologic findings. Radiographics 2001:21:403-17.

6 Cheuk W, Chan JK. Test and teach. Unusual pulmonary vascular pathology in a young dyspnoeic woman. Pulmonary tumour thrombotic microangiopathy (PTTM) resulting from diffuse-type adenocarcinoma of the stomach. Pathology 2001;33:504-7. 